\title{
Contrôle de croissance des ovins allaitants en Tunisie. I. Ajustement d'une courbe globale et analyse critique du protocole officiel
}

\author{
M. Ben Hamouda *
}

\begin{abstract}
Mots-clés
Ovin - Poids corporel - Gain de poids - Calcul - Contrôle de croissance - Tunisie.
\end{abstract}

\begin{abstract}
Résumé
Les données de contrôle de croissance de deux troupeaux expérimentaux de race ovine Barbarine, collectées durant 31 années, concernant 9642 agneaux contrôlés à la naissance et pesés ensuite à six reprises à 21 jours d'intervalle, ont été analysées. Les estimations obtenues grâce à l'ajustement d'une courbe de croissance globale ont été comparées aux évaluations faites dans le cadre du protocole conventionnel de contrôle de croissance «F2 ». Les fonctions de Brody, logistique, de Gompertz et de von Bertalanffy se sont convenablement ajustées à la série des sept contrôles. Les critères d'ajustement, coefficients de détermination $\left(\mathrm{R}^{2}\right)$ et sommes des carrés des écarts résiduels ont été estimés à 0,997 et 2,9,0,997 et 3,0,0,996 et 3,8, et 0,996 et 3,8 respectivement pour les fonctions de von Bertalanffy, Gompertz, Logistique et Brody. Les poids moyens à âge type, estimés par la méthode officielle, ont été de 3,4, 5,5, 9,1, $15,5,18,2$ et 22,2 kg respectivement à la naissance, à 10,30, 70, 90 et 120 jours d'âge. Le calcul des performances à âge type par la méthode conventionnelle s'est accompagné d'une faible perte de précision sur les poids de I'ordre de 5, 2, 0,8 et 0,5 p. 100 respectivement à 30, 70, 90 et 120 jours, et d'une baisse assez importante de 25, 19, 12 et 10 p. 100 respectivement des GMQ10/30 (gains moyens quotidiens entre 10 et 30 jours), GMQ30/70, GMQ30/90 et GMQ30/120. La perte de précision associée au GMQ10/30 a dépassé 50 p. 100 pour I'intervalle naissance - premier contrôle à 21 jours à cause d'une extrapolation inférieure, peu précise, du poids à 10 jours.
\end{abstract}

La population ovine de Tunisie compte aux alentours de 3,5 millions de femelles reproductrices (32). Elle est dominée par la Barbarine, race à queue grasse (65 p. 100), qui a développé, au cours des siècles, des capacités d'adaptation pour faire face aux aléas climatiques des régions méditerranéennes arides et semi-arides. L'amélioration génétique de cette population a été initiée à la fin des années 1960, avec l'appui de la coopération française, en adoptant l'approche française de contrôle de croissance mise au point par le Professeur Leroy à la fin des années 1950 et répertoriée, en Tunisie comme en France, comme méthode officielle (1, 17, $27,28)$. Sur la base de cette méthode, appelée également « protocole F2 », les différents bilans et travaux de recherche relatifs à la croissance des agneaux de race Barbarine, notamment, rapportent d'importantes variations non génétiques des performances $(4,6$, $7,11,12)$ qui sont souvent imputées aux conditions extensives de

\footnotetext{
* Institution de la recherche et de l'enseignement supérieur agricoles, 30 rue Alain Savary, 1002 Tunis, Tunisie.

Tél. : +21698385634; fax : +21671 796170

E-mail : m.b hammouda@iresa agrinet.tn
}

l'élevage ovin dans les zones arides et semi-arides de Tunisie sans jamais vérifier si la méthode de calcul elle-même est bien adaptée à ces données collectées dans un milieu très différent de celui dans lequel le protocole de contrôle a été élaboré. Dans cette perspective, discuter le principe de la méthode de calcul des performances à âge type incite à analyser la dynamique de la croissance des agneaux durant la période de contrôle (29), soit durant les quatre premiers mois de vie.

L'objectif de cette étude a été (a) d'ajuster une fonction non linéaire à la croissance globale, entre la naissance et environ 120 jours d'âge, (b) de comparer les performances à âge type obtenues par l'application du protocole F2 à celles déduites de la courbe de croissance la mieux ajustée, en espérant que cette dernière puisse atténuer les fluctuations à court terme, très prononcées en milieu aride et semi-aride, et (c) de préparer les éléments d'une analyse génétique permettant une éventuelle redéfinition des objectifs de sélection et des protocoles de contrôle des ovins allaitants en milieu difficile, alternatifs au protocole F2. Une attention particulière a été accordée aux qualités maternelles et à la valeur laitière des brebis, prédites par la croissance de leurs produits, car elles constituent le principal objectif des schémas de sélection à concevoir dans ces milieux méditerranéens $(5,31)$. 


\section{MATERIEL ET METHODES}

\section{Animaux}

Les données ont été collectées à la station expérimentale d'Oussletia de l'Institut national de la recherche agronomique de Tunisie (Inrat). Les animaux sont élevés en deux troupeaux dans les conditions extensives des régions semi-arides de la Tunisie centrale où la saison d'agnelage s'étend essentiellement sur les mois d'octobre et de novembre ; très peu d'agneaux naissent en septembre et en décembre. Entre 1968 et 1998, environ 12000 agneaux ont été contrôlés pour la croissance selon le protocole officiel de contrôle de croissance des ovins.

\section{Protocole officiel de pesée et de calcul}

Le protocole officiel consiste à peser, à partir du $21^{\mathrm{e}}$ jour après le début des agnelages et ensuite à intervalles réguliers d'environ 21 jours, tous les agneaux présents à chacune des six visites $(1,17,27,28)$. Pour l'ensemble des animaux des agnelages qui s'étalent sur deux mois, l'organisation de cinq à six chantiers de pesées sont en effet nécessaires pour le calcul des poids à âge type de tous ces agneaux : à 10 jours (P10), 30 jours (P30) et 70 jours d'âge (P70) ainsi que le gain moyen quotidien entre 10 et 30 jours (GMQ10/30) et entre 30 et 70 jours (GMQ30/70). Pour un animal particulier, ces quatre premières pesées sont suffisantes pour le calcul de ces critères. Le GMQ10/30 est un indicateur du potentiel d'allaitement de la mère, appelé par Ricordeau et Boccard « valeur laitière » (34), alors que le GMQ30/70 reflète davantage le potentiel de croissance précoce de l'agneau (17). Actuellement, dans les troupeaux expérimentaux, le contrôle est complété par une pesée à la naissance et la prise en compte de toutes les pesées permet aussi l'estimation du poids à 90 jours, voire à 120 jours pour les agneaux les plus âgés d'un lot d'agnelage. Le contrôle du poids à la naissance a été valorisé dans l'analyse du calcul du P10 et de la croissance initiale (GMQ00/30 et GMQ10/30). A partir de ces données, les situations suivantes ont été simulées :

A. Poids à la naissance ignoré ; c'est le cas des conditions réelles du contrôle de performances en ferme ; les poids à âge type sont estimés par interpolation lorsque le poids recherché est encadré par deux pesées. Dans le cas où celui-ci n'est pas encadré par deux pesées, il est alors estimé par extrapolation inférieure du poids à 10 jours des agneaux nés à moins de 10 jours avant la première visite.

B. Poids à la naissance contrôlé ; il est alors considéré comme un premier contrôle ; le poids à 10 jours, calculé dans le précédent scénario (A) par extrapolation inférieure est calculé par interpolation entre le poids à la naissance et la première pesée. L'indice « pn », affecté à une performance concernée par cette méthode de calcul, distingue le présent scénario du précédent. Nous pouvons logiquement supposer que le GMQ00/30 est estimé avec le maximum de fiabilité, car déduit du poids à la naissance mesuré le jour même de la naissance et du poids à 30 jours estimé à partir de deux pesées successives qui l'encadrent.

Pour des besoins d'analyses fines, de comparaison entre groupes d'agneaux dont l'une de leurs performances est strictement concernée par une méthode de calcul, l'indice " extrapolation inférieure » (ei), « interpolation » (in) ou « extrapolation supérieure » (es) est affecté à la performance concernée. Le dernier cas concerne les agneaux n'ayant pas atteint l'âge type recherché lors de la dernière visite $(70,90$ ou 120 jours pour le protocole F2 et 30 jours pour le protocole F1). L'extrapolation supérieure est effectuée dans la limite de la moitié de l'intervalle entre pesées séquentielles, soit 12 jours.
Dans les différentes situations, le calcul a été effectué selon la formule générale :

PAT $_{\mathrm{i}}=\mathrm{P}_{\mathrm{n}}+\left[\left(\mathrm{P}_{\mathrm{n}+1}-\mathrm{P}_{\mathrm{n}}\right) /\left(\mathrm{D}_{\mathrm{n}+1}-\mathrm{D}_{\mathrm{n}}\right)\right] *\left(\mathrm{at}_{\mathrm{i}}-\mathrm{D}_{\mathrm{n}}\right)$

où $\mathrm{PAT}_{\mathrm{i}}$ est le poids aux âges types $\left(\mathrm{at}_{\mathrm{i}}\right)$, soit $10,30,70,90$ et 120 jours, $D_{n}$ et $D_{n+1}$ sont les dates des contrôles cadrant l'âge type recherché at ${ }_{i}$, et $P_{n}$ et $P_{n+1}$ sont les poids aux contrôles $D_{n}$ et $D_{n+1}$.

Les GMQ00/30, GMQ10/30 ${ }_{\mathrm{pn}}$, GMQ10/30, GMQ30/70, GMQ30/90 et GMQ30/120 ont ensuite été calculés en utilisant le poids de naissance et les poids à âge type concernés : par exemple $\mathrm{GMQ} 10 / 30_{\mathrm{pn}}=\left(\mathrm{P} 30-\mathrm{P} 10_{\mathrm{pn}}\right) / 20$.

\section{Méthodes d'ajustement des courbes de croissance}

Plusieurs équations non linéaires représentant la croissance animale ont été développées $(14,20,21,22,33)$ et très souvent utilisées pour ajuster l'évolution du poids des ovins (2, 3, 7). Dans cette étude où le contrôle de croissance s'est limité aux quatre premiers mois de vie des agneaux, quatre équations ont été employées $(14,33)$ : (i) la fonction exponentielle dite de Brody $\left[\mathrm{Y}=\mathrm{A}\left(1-\mathrm{be} \mathrm{e}^{-\mathrm{kt}}\right)\right]$, et les fonctions sigmoïdes (ii) logistique $\left[\mathrm{Y}=\mathrm{A} /\left(1+\mathrm{be}^{-\mathrm{kt}}\right)\right]$, (iii) de Gompertz [Y $\left.=\mathrm{Ae}^{-\mathrm{e}(\mathrm{b}-\mathrm{kt})}\right]$, et (iv) de von Bertalanffy $\left[\mathrm{Y}=\mathrm{A}\left(1-\mathrm{e}^{(\mathrm{b}-\mathrm{kt})}\right)^{3}\right]$.

Les trois dernières admettent un point d'inflexion à $0,5,0,37$ et 0,29 de la valeur asymptotique A. La fonction de Richards, qui comporte quatre inconnues et un point d'inflexion variable, a été écartée à cause de son exigence en nombre de contrôles (25), notamment au niveau de la région où se situerait le point d'inflexion (13). Bilgin et coll. (8) ont également été contraints d'éliminer cette fonction de Richards dans leur étude sur la comparaison de plusieurs modèles d'ajustement de huit mesures de la circonférence testiculaire d'agneaux Awassi.

La qualité de l'ajustement a été appréciée par la somme des carrés des écarts résiduels (SCER) et le coefficient de détermination du modèle $\left(\mathrm{R}^{2}\right)$ non ajusté du fait que le nombre de paramètres des quatre fonctions était le même (trois) et que le nombre de pesées par agneau a été fixé à sept (poids à la naissance et six contrôles périodiques).

Les scénarios A et B du protocole conventionnel de contrôle de croissance ont permis une première analyse de la fiabilité du critère d'estimation de la valeur laitière à travers une comparaison des trois formes d'expression GMQ00/30, GMQ10/30 et GMQ10/30, la première étant prise comme référence du fait qu'elle est déduite d'une première mesure objective (poids à la naissance) et une seconde calculée systématiquement par interpolation à partir de deux pesées séquentielles. En outre, les performances estimées sur la courbe de croissance la mieux ajustée ont servi de références de comparaison de l'ensemble des performances à âge type, notamment celles non concernées par les scénarios de la méthode conventionnelle (P30, P70, P90, P120, GMQ30/70, GMQ30/90 et GMQ30/120).

Les différentes comparaisons ont été réalisées, de manière globale et en fonction de l'intervalle naissance - premier contrôle (tableau I), par l'application du modèle de régression linéaire suivant :

$\mathrm{Y}=\mathrm{a}+\mathrm{b} * \mathrm{X}+\mathrm{e}$

où $\mathrm{Y}$ sont les performances à âge type prises comme références de comparaison ; GMQ00/30, P10 et GMQ10/30 $\mathrm{B}$ du protocole officiel) ; P10, P30, $\mathrm{Pn} 70, \mathrm{P} 90, \mathrm{P} 120,{ }_{\mathrm{Gn}} \mathrm{MQ} 10 / 30$, GMQ30/70 et GMQ30/120 (courbe de croissance) ;

$\mathrm{X}$ représente toute performance à âge type, relative au protocole officiel autre que celle ayant servi de référence de comparaison, a 
l'ordonnée à l'origine, b le coefficient de régression, et e l'erreur aléatoire.

La perte de précision associée à l'estimation d'une performance, par rapport à celle prise comme référence, est appréciée par le terme $1-R^{2}, R^{2}$ étant le coefficient de détermination du modèle de

\section{Tableau I}

Effectif des agneaux de race Barbarine de la station Oussletia (Tunisie) nés entre 1968 et 1998 et ayant subi des contrôles de croissance, à la naissance et six fois à 21 jours d'intervalle, répartis en fonction de l'intervalle naissance - $1^{\text {er }}$ contrôle

\begin{tabular}{|c|c|}
\hline Intervalle naissance - $1^{\mathrm{er}}$ contrôle & Effectif \\
\hline 1 & - \\
\hline 2 & 1240 \\
\hline 3 & 678 \\
\hline 4 & 558 \\
\hline 5 & 563 \\
\hline 6 & 504 \\
\hline 7 & 447 \\
\hline 8 & 380 \\
\hline 9 & 341 \\
\hline 10 & 351 \\
\hline 11 & 382 \\
\hline 12 & 387 \\
\hline 13 & 454 \\
\hline 14 & 476 \\
\hline 15 & 533 \\
\hline 16 & 545 \\
\hline 17 & 490 \\
\hline 18 & 499 \\
\hline 19 & 468 \\
\hline 20 & 202 \\
\hline 21 & 144 \\
\hline Total & 9642 \\
\hline
\end{tabular}

régression (16), et complétée par le coefficient de régression b qui devrait avoisiner l'unité ; en effet, plus ce coefficient est proche de 1, plus le biais est indépendant de l'erreur aléatoire (9). En protocole officiel, l'extrapolation est déjà à l'origine d'un biais supplémentaire, par rapport à l'interpolation, la perte de précision qui lui est associée est alors qualifiée de «spécifique ». L'ajustement des fonctions de croissance individuelles et les régressions ont été exécutées respectivement par les procédures NLIN et REG du logiciel SAS 6.1 (35).

\section{RESULTATS}

\section{Statistiques générales}

Sur un total de 12000 agneaux contrôlés, le nombre de ceux qui possédaient les six pesées périodiques nécessaires à l'étude a été réduit à 9 642. Cependant, le poids à 120 jours n'a pu être estimé que pour 5854 agneaux. Le tableau II synthétise l'âge et le poids moyens aux différentes visites ainsi que le GMQ moyen entre contrôles successifs. Les agneaux de race Barbarine naissent avec un poids de l'ordre de $3,4 \mathrm{~kg}$ et atteignent environ $21 \mathrm{~kg}$ à 110 jours, âge moyen au sixième contrôle de croissance. Le GMQ entre contrôles successifs va en diminuant au fur et à mesure que l'agneau avance en âge ; en effet, d'une valeur dépassant les 200 $\mathrm{g} / \mathrm{j}$ en début de contrôle, le gain moyen quotidien chute à $130 \mathrm{~g} / \mathrm{j}$ en fin de période de contrôle. Le poids à 10 jours, quand il est estimé par interpolation entre le $\mathrm{PN}$ et la première pesée, subit les conséquences de la variabilité de cette dernière, très élevée durant la première semaine de vie d'un agneau.

\section{Qualité de l'ajustement d'une fonction non linéaire}

Les critères d'appréciation de la qualité de l'ajustement des fonctions non linéaires à la croissance pondérale des agneaux de race Barbarine sont détaillés dans le tableau III. Les fonctions de Gompertz et de von Bertalanffy ont donné les coefficients de détermination les plus élevés ; ceux relatifs aux fonctions logistique et de Brody ont été significativement plus faibles $(\mathrm{P}<0,05)$. L'analyse de la SCER a précisé encore davantage la comparaison dans la mesure où elle a permis de départager les fonctions Gompertz et Bertalanffy en faveur de cette dernière qui a donné la valeur la plus faible $(\mathrm{P}<0,05)$. Par ailleurs, l'examen des paramètres des différentes fonctions (tableau III) a permis de constater que la valeur asymptotique « $\mathrm{A} »$ a été estimée, par les quatre fonctions, avec

\section{Tableau II}

Age, poids et GMQ des agneaux de race Barbarine de la station Oussletia (Tunisie) aux différents contrôles de croissance effectués entre 1968 et 1998 selon le protocole officiel

\begin{tabular}{|c|c|c|c|c|c|c|}
\hline & \multicolumn{2}{|c|}{ Age } & \multicolumn{2}{|c|}{ Poids (kg) } & \multicolumn{2}{|c|}{ GMQ (g/j) } \\
\hline & Moyenne & Ecart-type & Moyenne & Ecart-type & Moyenne & Ecart-type \\
\hline Naissance & - & - & 3,4 & 0,7 & - & - \\
\hline $1^{\mathrm{er}}$ contrôle & 10,2 & 6,1 & 5,5 & 1,8 & $213^{*}$ & $156^{*}$ \\
\hline $2^{\mathrm{e}}$ contrôle & 30,2 & 6,2 & 9,1 & 2,7 & 181 & 78 \\
\hline $3^{\mathrm{e}}$ contrôle & 52,2 & 6,3 & 12,5 & 3,6 & 170 & 73 \\
\hline $4^{\mathrm{e}}$ contrôle & 70,4 & 6,3 & 15,6 & 4,4 & 151 & 76 \\
\hline $5^{\mathrm{e}}$ contrôle & 90,9 & 6,6 & 18,3 & 5,2 & 131 & 52 \\
\hline $6^{\mathrm{e}}$ contrôle & 111,5 & 7,3 & 20,9 & 6,0 & 129 & 91 \\
\hline
\end{tabular}

GMQ : gain de poids moyen quotidien

* La variabilité du GMQ naissance - $1^{\mathrm{er}}$ contrôle est surestimée par les valeurs relatives aux faibles intervalles (inférieures à 6 jours), leur élimination ramène la moyenne et l'écart-type respectivement à 206 et 93. 
une variabilité assez élevée ; le coefficient de variation a été de l'ordre de l'unité pour les quatre fonctions.

\section{Performances à âge type}

Les performances moyennes aux différents âges types sont présentées dans le tableau IV. En se référant à la méthode conventionnelle, les agneaux de l'étude ont pesé en moyenne 5,5, 9,1, 15,5, 18,2 et $22,2 \mathrm{~kg}$, respectivement à $10,30,70,90$ et 120 jours d'âge. Le GMQ a connu une diminution au fur et à mesure que l'agneau avançait en âge ; il a été en moyenne de 180,161, 152 et $145 \mathrm{~g} / \mathrm{j}$, respectivement pour les périodes $10-30,30-70,30-90$ et 30-120. Le fait de considérer le poids à la naissance comme un premier contrôle a permis d'estimer un GMQ10/30 pà $184 \mathrm{~g} / \mathrm{j}$ et un GMQ00/30 à $190 \mathrm{~g} / \mathrm{j}$. Par ailleurs, la fonction de von Bertalanffy a donné, pour les mêmes performances, des valeurs significativement différentes $(\mathrm{P}<0,05)$, légèrement inférieures à 10 et 30 jours (respectivement 5,23 et $8,97 \mathrm{~kg}$ ) et supérieures à 70 et 90 jours (respectivement 15,66 et
$18,30 \mathrm{~kg}$ ). Le GMQ10/30 estimé par la fonction von Bertalanffy n'a pas été significativement différent du GMQ10/30 ${ }_{\mathrm{pn}}(\mathrm{P}>0,05)$. Il en a été de même pour le GMQ30/90, pour lequel les deux estimations n'ont pas été significativement différentes. Les deux méthodes ont donné, en moyenne, un GMQ avant 30 jours d'âge (GMQ00/30, GMQ10/30 ${ }_{\mathrm{pn}}$ et GMQ10/30) supérieur au GMQ30/90. Cependant, la représentation par année de naissance (figure 1) a révélé qu'il ne s'agissait pas d'une règle mais d'une tendance générale qui s'est inversée pendant certaines années (1984 et 1988, par exemple).

\section{Pertes de précision associées au calcul des performances à âge type}

\section{Cas du protocole conventionnel}

Les résultats des comparaisons permises par les scénarios du protocole conventionnel sont montrés dans le tableau V. Le GMQ10/30, comparé au GMQ00/30, a été accompagné de la perte de précision moyenne la plus élevée $\left(1-\mathrm{R}^{2}=24,8\right.$ p. $\left.100 ; \mathrm{b}=0,802\right)$. L'origine

\section{Tableau III}

Paramètres des fonctions non linéaires ajustées à la croissance des agneaux de race Barbarine et critères d'appréciation de la qualité de l'ajustement

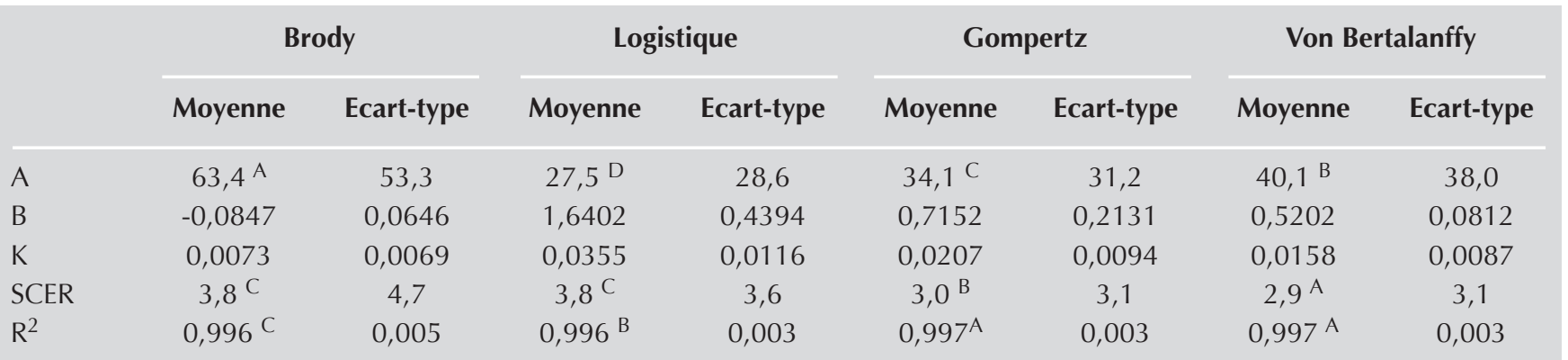

Sur une même ligne, les moyennes portant des lettres différentes sont significativement différentes $(\mathrm{P}<0,05)$

SCER : somme des carrés des écarts résiduels

Tableau IV

Performances à âge type des agneaux de race Barbarine calculées par la méthode officielle ou estimées par l'ajustement de la fonction de von Bertalanffy

Protocole officiel «F2»

\begin{tabular}{|c|c|c|c|c|c|}
\hline \multirow[b]{2}{*}{ Performance } & \multirow[b]{2}{*}{ Effectif } & \multicolumn{2}{|c|}{ Protocole officiel « F2 » } & \multicolumn{2}{|c|}{$\begin{array}{l}\text { Ajustement de la fonction } \\
\text { de von Bertalanffy }\end{array}$} \\
\hline & & Moyenne & Ecart-type & Moyenne & Ecart-type \\
\hline PN & 9642 & $3,39^{\mathrm{B}}$ & 0,69 & $3,50^{A}$ & 0,78 \\
\hline $\mathrm{P} 10_{\mathrm{pn}}$ & 9642 & $5,39^{A}$ & 1,21 & $5,23^{\mathrm{B}}$ & 1,14 \\
\hline P10 & 9642 & $5,48^{\mathrm{A}}$ & 1,34 & $5,23^{\text {в }}$ & 1,14 \\
\hline P30 & 9642 & $9,09^{A}$ & 2,37 & $8,97^{\text {В }}$ & 2,29 \\
\hline P70 & 9642 & $15,53^{B}$ & 4,19 & $15,66^{\mathrm{A}}$ & 4,17 \\
\hline P90 & 9642 & $18,17^{\mathrm{A}}$ & 4,95 & $18,30^{A}$ & 4,96 \\
\hline P120 & 5854 & $22,16^{\mathrm{A}}$ & 6,17 & $21,55^{\mathrm{B}}$ & 6,16 \\
\hline GMQ00/30 & 9642 & $190^{\mathrm{A}}$ & 67 & $182^{B}$ & 65 \\
\hline $\mathrm{GMQ} 10 / 30_{\mathrm{pn}}$ & 9642 & $185^{A}$ & 72 & $187^{A}$ & 66 \\
\hline GMQ10/30 & 9642 & $180^{\mathrm{B}}$ & 69 & $187^{\mathrm{A}}$ & 66 \\
\hline GMQ30/70 & 9642 & $161^{\mathrm{B}}$ & 57 & $167^{A}$ & 54 \\
\hline GMQ30/90 & 9642 & $152^{\mathrm{A}}$ & 52 & $152^{A}$ & 53 \\
\hline GMQ30/120 & 5854 & $145^{\mathrm{A}}$ & 51 & $137^{B}$ & 51 \\
\hline
\end{tabular}

$\mathrm{P}$ : poids ; PN : poids à la naissance ; GMQ : gain de poids moyen quotidien

Sur une même ligne, les moyennes portant des lettres différentes sont significativement différentes $(\mathrm{P}<0,05)$ 
Tableau V

Critères d'appréciation (pertes de précision $1-R^{2}$ et coefficient de régression $b$ ) de la différence de précision de calcul des performances à âge type des agneaux de race Barbarine par les différents scénarios du protocole officiel

\begin{tabular}{|c|c|c|c|c|c|c|}
\hline \multirow[t]{2}{*}{ Performance/référence } & \multicolumn{2}{|c|}{ Performance globale } & \multicolumn{2}{|c|}{ Interpolation de P10 } & \multicolumn{2}{|c|}{$\begin{array}{c}\text { Extrapolation } \\
\text { inférieure de P10 }\end{array}$} \\
\hline & $1-R^{2}$ & $\mathbf{b}$ & $1-R^{2}$ & b & $1-R^{2}$ & b \\
\hline $\mathrm{P} 10 / \mathrm{P} 10_{\mathrm{pn}}$ & 8,6 & 0,863 & 0,0 & 1,000 & 13,5 & 0,758 \\
\hline $\mathrm{P} 30 / \mathrm{P} 30_{\mathrm{es}}$ & 1,7 & 0,954 & 2,9 & 0,920 & 0,0 & 1,000 \\
\hline $\mathrm{P} 70 / \mathrm{P} 70_{\mathrm{es}}$ & 0,8 & 0,983 & 1,5 & 0,968 & 0,0 & 1,000 \\
\hline $\mathrm{P} 90 / \mathrm{P} 90_{\mathrm{es}}$ & 0,5 & 0,987 & 0,9 & 0,977 & 0,0 & 1,000 \\
\hline GMQ10/30 / GMQ10/30 & 7,4 & 0,921 & 0,0 & 1,000 & 13,5 & 0,854 \\
\hline GMQ10/30 / GMQ10/30 es & 4,3 & 1,042 & 7,6 & 1,084 & 0,0 & 1,000 \\
\hline GMQ10/30 / GMQ00/30 & 24,8 & 0,802 & 14,1 & 0,895 & 33,9 & 0,718 \\
\hline $\mathrm{GMQ} 10 / 30_{\mathrm{pn}} / \mathrm{GMQ} 00 / 30$ & 12,2 & 0,905 & 14,1 & 0,895 & 8,7 & 0,919 \\
\hline $\mathrm{GMQ00/30/GMQ00/30}$ es & 2,3 & 0,943 & 5,8 & 0,902 & 0,0 & 1,000 \\
\hline GMQ30/70 / GMQ30/70 es & 2,6 & 0,945 & 4,4 & 0,911 & 0,0 & 1,000 \\
\hline GMQ30/90 / GMQ30/70 es & 1,2 & 0,962 & 2,7 & 0,937 & 0,0 & 1,000 \\
\hline
\end{tabular}

$P$ : poids ; GMQ : gain de poids moyen quotidien

de cette perte de précision serait imputée à l'estimation de P10; en effet, un taux de 14,1 p. 100 a été associé à $\mathrm{P} 10_{\text {in }}$ (calculé par interpolation) et de 33,9 p. 100 à $\mathrm{P}^{10}{ }_{\mathrm{ei}}$ (estimé par extrapolation inférieure), ce dernier étant qualifié de perte de précision spécifique. Présentée en fonction de l'intervalle entre la naissance et le premier contrôle (figure 2), la perte de précision associée à $\mathrm{P} 10$ a atteint 35 p. 100 à 21 jours alors que celle affectant le GMQ10/30, au même intervalle, a dépassé 60 p. 100 . A une extrapolation supérieure de $\mathrm{P} 30$, cas où l'agneau aurait deux contrôles uniquement, est associée 1,7 p. 100 de perte de précision globale et 2,9 p. 100 de perte de précision spécifique entraînant une perte de précision sur le GMQ10/30 estimée respectivement à 4,3 et 7,6 p. 100 de manière globale et spécifique (tableau V). En cas d'extrapolation supérieure de $\mathrm{P} 70$, la perte de précision globale et spécifique, associée à cette performance, a été respectivement de 0,8 et 1,5 p. 100, et de 2,6 et 4,4 p. 100 sur le GMQ30/70 (tableau V).

\section{Performances estimées par la courbe de croissance}

La perte de précision associée au calcul des performances à âge type par la méthode conventionnelle, par rapport à celles estimées par la courbe de croissance ajustée par la fonction de von Bertalanffy, est montrée dans le tableau VI. Le poids à la naissance estimé par la courbe de croissance a été comparé au réel ; ainsi, la perte de précision associée à 35,2 p. 100 a été élevée.

Les pertes de précision associées aux GMQ10/30, GMQ10/30pn et P10 par rapport au GMQ10/30 et P10 estimés par l'ajustement d'une fonction von Bertalanffy sont présentées à la figure 3. A un P10 estimé par extrapolation inférieure a été associée une perte de précision égale à 17 p. 100 , celle associée au GMQ10/30 a été de 26,3 p. 100 ; représentée en fonction de l'intervalle naissance - premier contrôle, cette dernière a évolué entre 10 p. 100 à 2 jours d'intervalle et 50 p. 100 à 21 jours. Le contrôle du poids à la naissance a atténué la perte de précision associée à $\mathrm{P} 10_{\mathrm{pn}}$ à un taux de 6,5 p. 100. Par ailleurs, les pertes de précision associées au calcul du GMQ00/30 et du GMQ10/30 ont été comparables (respectivement 19,7 et 20,0 p. 100) ; le coefficient de régression sur le GMQ00/30 a été plus élevé $(0,916)$ que celui de la régression sur le GMQ10/30 ${ }_{\text {pn }}(0,845)$.

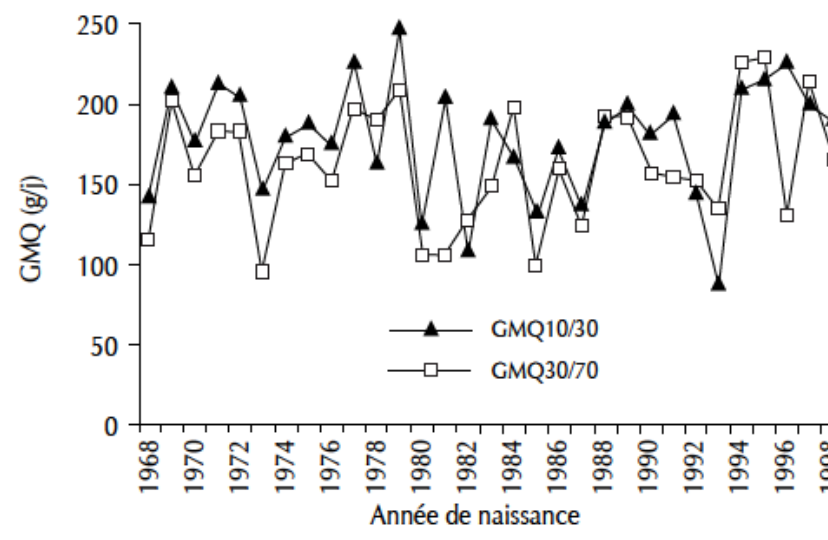

Figure 1 : variation des CMQ10/30 (gain moyen quotidien entre 10 et 30 jours) et GMQ30/70 des agneaux de race Barbarine en fonction de l'année de naissance.

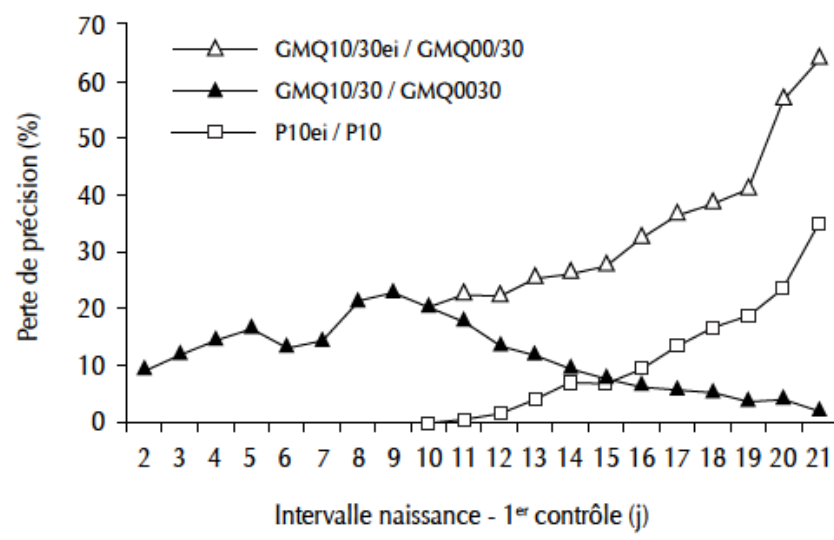

Figure 2 : perte de précision associée aux GMQ10/30 (gain moyen quotidien entre 10 et 30 jours), GMQ10/30ei et P10ei respectivement par rapport au GMQ00/30 et P10pn (ei : extrapolation inférieure de $P 10$; pn : le premier contrôle est celui du poids à la naissance). 
Les poids à $30,70,90$ et 120 jours ont été calculés avec une perte de précision respectivement de $5,3,1,9,0,8$ et 0,5 p. 100 . Ces pertes de précision se sont conjuguées au niveau du GMQ pour donner un taux de $18,6,12,3$ et 10,0 p. 100 , respectivement pour les périodes $30-70,60-90$ et $30-120$ jours. L'évolution des pertes de précision associées au calcul des performances calculées par la méthode F2 par rapport à celles estimées par l'ajustement d'une fonction von Bertalanffy est présentée à la figure 4 .

\section{- DISCUSSION}

Les fonctions non linéaires de von Bertalanffy, de Gompertz, logistique et de Brody se sont ajustées relativement bien aux données de contrôle de croissance des agneaux de race Barbarine, collectées durant leurs cent vingt premiers jours de vie. Le meilleur ajustement, jugé par le coefficient de détermination, a été obtenu par la fonction de von Bertalanffy, suivie par la fonction de Gompertz. Par ailleurs, le fait que la valeur asymptotique n'ait pas été estimée avec une précision suffisante ne remet pas en cause la qualité de l'ajustement mais signifierait que cette dernière concerne uniquement la période couverte par le contrôle de croissance qui est relativement trop courte pour pouvoir estimer le poids adulte avec une précision suffisante. Goliomytis et coll. (15) pouvaient exiger une précision de la prédiction du poids adulte (valeur asymptotique) du moment qu'ils avaient contrôlé les animaux jusqu'à l'âge de 720 jours.

Les présents résultats sont comparables à ceux rapportés par Topal et coll. (36) qui ont conclu que la fonction de Gompertz s'ajuste mieux à la croissance des agneaux Morkaraman alors que la fonction de von Bertalanffy s'ajuste mieux à la croissance des agneaux Awassi. Lambe et coll. (23) rapportent, pour un coefficient de détermination moyen supérieur à 0,979 , que les différents modèles (Gompertz, logistique, Richards et exponentiel) s'ajustent bien à la croissance des agneaux. Certains auteurs n'ont pas trouvé

\section{Tableau VI}

Critères $\mathrm{d}^{\prime}$ appréciation de la différence de précision (pertes de précision $1-\mathrm{R}^{2}$ et coefficient de régression

b) du calcul des performances à âge type des agneaux de race Barbarine par le protocole officiel et par I'ajustement d'une fonction de von Bertalanffy

\begin{tabular}{lcc} 
& $\mathbf{1 - \mathbf { R } ^ { \mathbf { 2 } }}$ & $\mathbf{b}$ \\
\hline PN & 35,2 & 0,705 \\
P10 & & 1,024 \\
P10 & 6,5 & 0,777 \\
P30 & 17,0 & 0,941 \\
P70 & 5,3 & 0,987 \\
P90 & 1,9 & 0,994 \\
P120 & 0,8 & 0,976 \\
GMQ00/30 & 0,5 & 0,916 \\
GMQ10/30 & 19,7 & 0,845 \\
GMQ10/30 & 20,0 & 0,777 \\
GMQ30/70 & 26,3 & 0,861 \\
GMQ30/90 & 18,6 & 0,659 \\
GMQ30/120 & 12,0 & 0,932
\end{tabular}

$P$ : poids ; PN : poids à la naissance ; GMQ10/30 : gain de poids moyen quotidien entre 10 et 30 jours indispensable une comparaison entre fonctions et ont plutôt opté pour l'une d'entre elles : par exemple, la fonction de Brody pour ajuster la croissance des agneaux Mehraben d'Iran $(2,3)$ ou des bovins Angus (19), et la fonction de Gompertz pour étudier la croissance des poulets (26).

Les performances de croissance des ovins allaitants en Tunisie, de race Barbarine en l'occurrence, calculées par la méthode officielle ou par l'ajustement de la fonction de von Bertalanffy, retenue dans la dernière partie de cette étude, ont été fortement influencées par le milieu d'élevage à dominance aride et semi-aride. Le GMQ a diminué au fur et à mesure que l'agneau a avancé en âge. Le GMQ10/30 a été le plus souvent supérieur au GMQ30/70 et à plus forte raison au GMQ30/90 ou encore au GMQ30/120. Ceci rejoint les résultats de plusieurs travaux de recherche $(4,5,6,7$, 11,12 ) ou de recherche développement (32). Ce dernier travail sur les ovins tunisiens rapporte un GMQ10/30 de 165 et $145 \mathrm{~g} / \mathrm{j}$ respectivement pour les mâles et les femelles contre 121 et $106 \mathrm{~g} / \mathrm{j}$ de GMQ30/70 pour les deux mêmes sexes.

Ces différences ne constituent pas des caractéristiques raciales mais reflètent plutôt l'influence du milieu et le caractère extensif de l'élevage ovin en Tunisie. En effet, les besoins alimentaires d'un agneau sont mieux assurés sous sa mère que lorsqu'il est livré à lui-même pour chercher son alimentation sur les parcours des régions arides et semi-arides du Sud et du Centre tunisien.

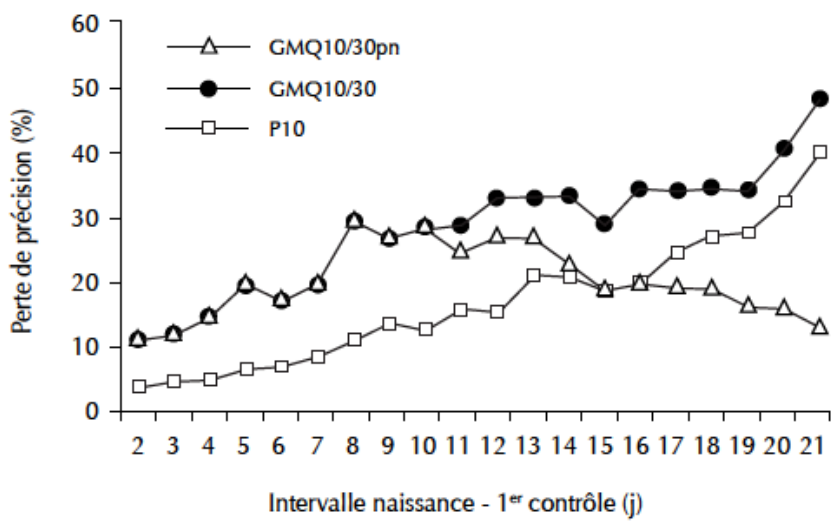

Figure 3 : perte de précision associée aux GMQ10/30 (gain moyen quotidien entre 10 et 30 jours), GMQ10/30pn et $P 10$ par rapport aux GMQ10/30 et P10 estimés par l'ajustement d'une fonction de von Bertalanffy.

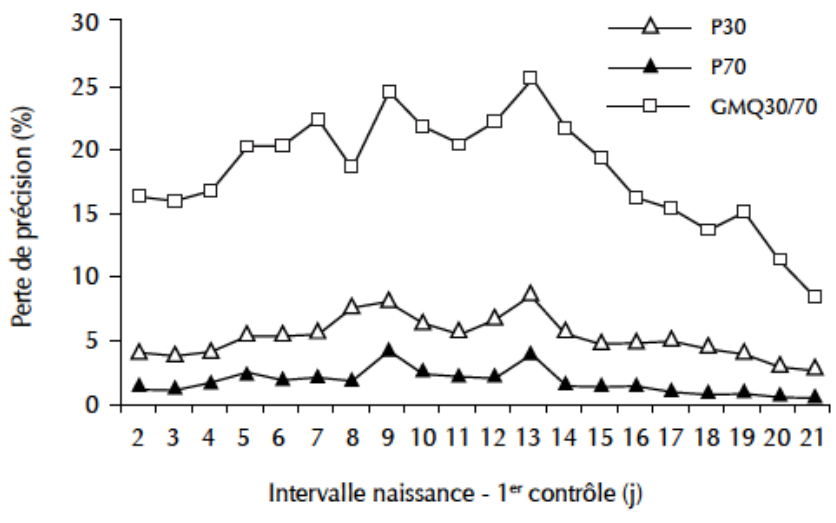

Figure 4 : perte de précision associée au calcul des performances calculées par le protocole conventionnel par rapport à celles estimées par l'ajustement d'une fonction de von Bertalanffy. 
Le même comportement a été observé dans d'autres régions du monde. Chikhi et Boujenane (10) rapportent un GMQ moyen de 213 et $190 \mathrm{~g} / \mathrm{j}$, respectivement pour les périodes $00 / 30$ et 30/70 jours, chez la race Boujaâd au Maroc. En Martinique, le GMQ10/30 des agneaux était plus élevé que le GMQ30/70 aussi bien en bergerie que sur pâturage, avec respectivement 175 et $147 \mathrm{~g} / \mathrm{j}$ contre 137 et $120 \mathrm{~g} / \mathrm{j}$ (24). Rekik et Ben Hamouda (31) ont illustré l'effet du milieu sur le GMQ des ovins en Tunisie par un gradient Nord - Sud et Nord - Sud-Ouest selon lequel le ratio GMQ30/70 / GMQ10/30, positif au Nord, change de signe et augmente en valeur absolue en avançant dans le sens du gradient.

Cette situation n'est pas sans conséquence sur l'application du protocole officiel de contrôle de croissance des ovins allaitants mis au point en France, dans des conditions favorables à une croissance plus régulière $(18,29)$, du fait d'une meilleure couverture des besoins des animaux et de la maitrise des techniques d'élevage. En effet, les fluctuations du poids, mesurées lors du contrôle de croissance, sont à l'origine d'un certain taux d'erreur qui accompagne le calcul des poids à âge type ; ces taux sont amplifiés au niveau du calcul des GMQ à cause de la conjugaison des deux taux d'erreur associés à chacun des poids à âge type servant à leur calcul.

Sur le calcul des poids, le taux d'erreur, exprimé en perte de précision $\left(1-\mathrm{R}^{2}\right)$, le plus élevé a été celui associé au calcul de P10 par extrapolation inférieure. En régions semi-arides de la Tunisie centrale, les variations climatiques inter et intra-années sont très prononcées et les mauvaises années se répercutent sur la croissance des agneaux qui a, le plus souvent, tendance à ralentir.

Avec ce ralentissement de la croissance précoce, l'estimation du P10 par extrapolation inférieure perd de la précision (figure 5) et a tendance à surestimer la valeur du poids. La perte de précision sur P10 se répercute évidemment sur le calcul du GMQ10/30 pour dépasser le taux de 50 p. 100 chez les agneaux pesés une première fois à 21 jours et une seconde fois à 42 jours d'âge. En outre, une surestimation de P10 signifie une sous-estimation du GMQ10/30. Le cas 3 de la figure 5 , situation extrême, représente une perte de poids au second contrôle et un GMQ10/30 négatif. Naves et coll. (27) ont rapporté un poids à 10 jours des chevreaux créoles plus élevé par extrapolation inférieure, par rapport à une interpolation entre PN et P1. Les mêmes auteurs ont rapporté aussi un GMQ10/30 supérieur au GMQ30/70, soit $90 \mathrm{~g} / \mathrm{j}$ contre $74 \mathrm{~g} / \mathrm{j}$, ce qui semble cohérent et corroborer les présents résultats. Au Maroc, le protocole de contrôle de croissance envisage, chaque fois que c'est possible, une pesée des agneaux à la naissance (1) ; la valeur

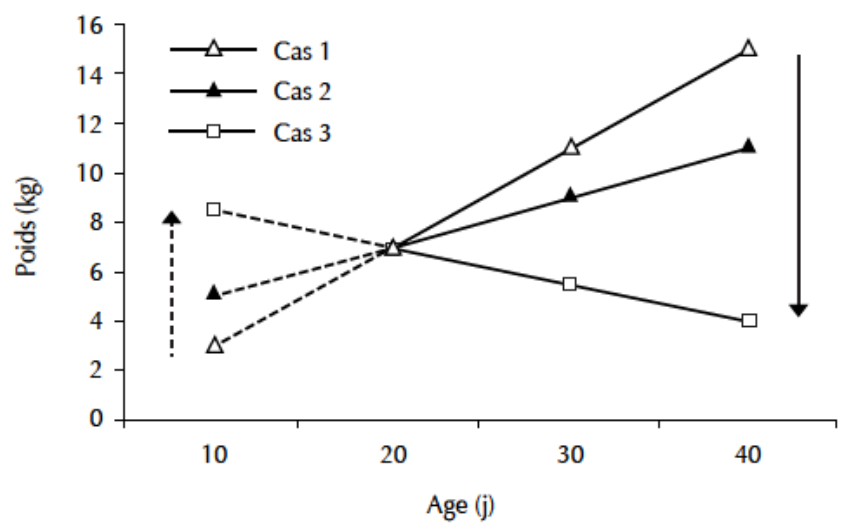

Figure 5 : influence du poids au deuxième contrôle sur l'estimation du P10 calculé par extrapolation inférieure ; un ralentissement de la croissance entre le premier et le second contrôle se traduit par une surestimation de P10. laitière est, dans ces conditions, exprimée en GMQ00/30 plutôt qu'en GMQ10/30 (10).

Les pertes de précision associées à l'extrapolation supérieure de P30 pour les agneaux ayant deux contrôles, d'une part, et de P70 pour les agneaux ayant subi quatre contrôles, d'autre part, ont été peu importantes. Les deux approches d'estimation de la perte de précision, associée au calcul du GMQ10/30, ont donné des résultats comparables en valeur moyenne $(25$ p. 100$)$ et en termes de variation de cette perte de précision en fonction de l'intervalle naissance - premier contrôle (figure 6), ce qui valide la cohérence des hypothèses de travail et des résultats obtenus. En outre, la comparaison à la courbe de croissance a permis de classer les trois expressions de la valeur laitière, GMQ00/30, GMQ10/30 et GMQ10/30 auxquelles sont associées des taux de perte de précision de $19,7,0,20$ et 25 p. 100. Le GMQ00/30 du protocole officiel est évidemment plus précis que celui estimé par la courbe de croissance ajustée du fait que le poids à la naissance est estimé sur la courbe avec une perte de précision égale à 35,2 p. 100 (tableau VI). L'avantage de la courbe de croissance se situerait plutôt au niveau de la seconde phase de la période de contrôle (30/90, voire $30 / 120$ jours) pour fournir un ordre de grandeur de la perte de précision associée au calcul des performances à âge type relatives à cette phase. Il faudrait par prudence, parler plutôt, de différences de précision que de perte de précision, du fait que l'ajustement d'une courbe de croissance amortit des variations, à court terme, auxquelles la méthode officielle est plus sensible.

\section{CONCLUSION}

La croissance des agneaux de race Barbarine, durant la période de contrôle officiel, s'est bien ajustée par une fonction sigmoïde (logistique, de Gompertz et de von Bertalanffy), voire par une fonction exponentielle (Brody). Toutefois, la fonction de von Bertalanffy a présenté les meilleurs critères d'ajustement. Les poids à âge type peuvent alors être estimés par la courbe de croissance ajustée. Quel que soit le mode de calcul, le GMQ des agneaux de race Barbarine subit un ralentissement assez sévère sous l'effet des conditions difficiles d'élevage dans les régions arides et semiarides de Tunisie ; il diminue au fur et à mesure que l'agneau avance en âge. Aussi, le protocole conventionnel qui suppose une linéarité de la croissance ne semble pas le mieux approprié à de telles situations, notamment pour l'estimation de la valeur laitière.

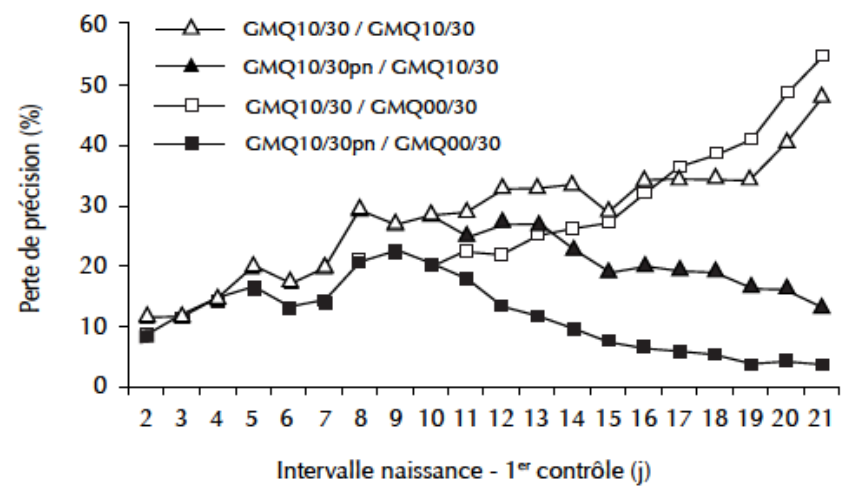

Figure 6 : perte de précision associée au calcul du CMQ10/30 (gain moyen quotidien entre 10 et 30 jours) et du GMQ10/30pn par rapport au GMQ10/30 estimé par l'ajustement d'une fonction de von Bertalanffy et du GMQ0O/30 relatif au protocole conventionnel (pn : poids à la naissance). 
La perte de précision associée au calcul du GMQ10/30, en moyenne égale à 25 p. 100 , peut dépasser le taux de 50 p. 100 pour des agneaux ayant subi le premier contrôle à 21 jours d'âge. Ce taux particulièrement élevé est dû à une mauvaise estimation du poids à 10 jours par extrapolation inférieure, fortement déterminée par le poids au second contrôle. Les pertes de précision sur l'estimation de P30, P70, P90 et P120 étaient faibles, égales en moyenne respectivement à $5,2,0,8$ et 0,5 p. 100 mais se traduisaient par une perte de précision plus élevée sur le GMQ (19, 12 et 10 p. 100 de pertes associées aux GMQ30/70, GMQ30/90 et GMQ30/120).

Des rectifications de la méthode de calcul des performances à âge type des agneaux, en milieu difficile, s'imposent du fait que la perte de précision la plus élevée est associée à la valeur laitière qui constituerait le principal critère de sélection dans de telles conditions. Une analyse génétique permettrait de décider si la révision doit se limiter au protocole de contrôle ou si, au contraire, elle doit aller en profondeur pour adopter de nouveaux objectifs de sélection mieux adaptés au milieu et aux systèmes de production de la rive sud de la Méditerranée.

\section{BIBLIOGRAPHIE}

1. AIT BIHI N., BOUJENANE I., 1997. Contrôle de performances des ovins en milieu difficile : cas du Maroc. Cah. Options méditerr. Sér. A, $33: 11-34$.

2. BATHAEI S.S., LEROY P.L., 1996. Growth and mature weight of Mehraban Iranian fat-tailed sheep. Small Rumin. Res., 22: 155-162.

3. BATHAEI S.S., LEROY P.L., 1998. Genetic and phenotypic aspects of the growth curve characteristics in Mehraban Iranian fat-tailed sheep. Small Rumin. Res., 29: 261-269.

4. BEDHIAF ROMDHANI S., DJEMALI M., 2006. New genetic parameters to exploit genetic variability in low input production systems. Livest. Sci., 99: 119-123.

5. BEN GARA A., 2000. Définition des objectifs de sélection des ovins de race Barbarine en Tunisie. Cah. Options méditerr. Sér. A, 43 : 111-116.

6. BEN GARA A., ROUISSI H., JURADO J.J., BODIN L., GABINA D., BOUJENANE I., MAVROGENIS A.P., DJEMALI M., SERRADILLA J.M., 1997. Etude de la simplification du protocole de pesées chez les ovins à viande. Cah. Options méditerr. Sér. A, $33:$ 11-34.

7. BEN HAMOUDA M., 1985. Description biométrique et amélioration génétique de la croissance pondérale des ovins de race Barbarine. Thèse Doct. Sci. Agron., Université de Gand, Belgique, $166 \mathrm{p}$.

8. BILGIN O.C., EMSEN E., DAVIS M.E., 2004. Comparison of non-linear models for describing the growth of scrotal circumference in Awassi male lambs. Small Rumin. Res., 52 : 155-160.

9. BOULOC N., BARILLET F., BOICHARD D., SIGWALD J.P., BRIDOUX G., 1991. Etude des possibilités d'allègement du contrôle laitier officiel chez les caprins. Ann. Zootech., 40 : 125-139.

10. CHIKHI A., BOUJENANE I., 2003. Performances de reproduction et de production des ovins de race Boujaâd au Maroc. Rev. Elev. Méd. vét. Pays trop., $\mathbf{5 6}:$ 83-88.

11. DJEMALI M., ALOULOU R., BEN SASSI M., 1994. Adjustment factors and genetic and phenotypic parameters for growth traits of Barbarine lambs in Tunisia. Small Rumin. Res., 13: 41-47.

12. DJEMALI M., JMAL S., HAMMAMI H., BEN DHIAF S., CHALLAH A., 1995. Acquis de la recherche en matière d'évaluation génétique des ovins et des caprins en Tunisie. Cah. Options méditerr., 11 : 173-184.

13. DOREN P.E., BAKER J.F., LONG C.R., CATRWRIGHT T.C., 1989 Estimating parameters of growth curves of bulls. J. Anim. Sci., 67: 14321445

14. FITZHUGH H.A. JR, 1976. Analyses of growth curves and strategy for altering their shape. J. Anim. Sci., 42: 1036-1051.

15. GOLIOMYTIS M., ORFANOS S., PANOPOULOU E., ROGDAKIS E., 2005. Growth curves for body weight and carcass components, and carcass composition of the Karagouniko sheep, from birth to 720 days of age. Small Rumin. Res., 66: 222-229.
16. GONZALO C., OTHMANE M.H., FUERTES J.A., DE LA FUENTE L.F., SAN PRIMITIVO F., 2003. Losses of precision associated with simplified designs of milk recording for dairy ewes. J. Dairy Res., 70: 441-444.

17. INRA / INSTITUT DE L'ELEVAGE, 1995. Répertoire français des méthodes et procédures de contrôle et d'évaluation génétique des reproducteurs ovins et bovins de races allaitantes. Paris, France, Institut de l'élevage, $105 \mathrm{p}$.

18. JULLIEN E., GRIFFON L., LEMAIRE V., TIPHINE L., 2006. Bilan de contrôle de performances d'ovins allaitants "Campagne 2005 ». Paris, France, Institut de l'élevage, 103 p.

19. KAPS M., HERRING W.O., LAMBERSON W.R., 2000. Genetic and environmental parameters for traits derived from the Brody growth curve and their relationships with weaning weight in Angus cattle. J. Anim. Sci., 78: 1436-1442.

20. KEBREAB E., SCHULIN-ZEUTHEN M., LOPEZ S., SOLER J., DIAS R.S., DE LANGE C.F.M., FRANCE J., 2007. Comparative evaluation of mathematical functions to describe growth and efficiency of phosphorus utilization in growing pigs. J. Anim Sci., 85: 2498-2507.

21. KNIZETOVA H., HYANEK J., HYANKOVA L., BELICEK P., 1995. Comparative study of growth curves in poultry. Genet. Sel. Evol., 27: 365-375.

22. KOHN F., SHARIFI A.R., SIMIANER H., 2007. Modelling the growth of the Goettingen minipig. J. Anim. Sci., 85: 84-92.

23. LAMBE N.R., NAVAJAS E.A., SIMM G., BUNGER L., 2006. A genetic investigation of various growth models to describe growth of lambs of two contrasting breeds. J. Anim. Sci., 84: 2642-2654.

24. MAHIEU M., AUMONT G., ALEXANDRE G., 1997. Elevage intensif des ovins tropicaux en Martinique. Prod. Anim., 10 : 21-32.

25. MIGNON-GRASTEAU S., BEAUMONT C., 2000. Les courbes de croissance chez les oiseaux. Prod. Anim., 13 : 337-348.

26. MIGNON-GRASTEAU S., PILES M., VARONA L., DE ROCHAMBEAU H., POIVEY J.P., BLASCO A., BEAUMONT C., 2000. Genetic analyses of growth curve parameters for male and female chickens resulting from selection on shape of growth curve. J. Anim. Sci., 78: 2515-2524.

27. NAVES M., MENENDEZ BUXADERA A., ALEXANDRE G., MANDONNET N., 2001. Etude comparative sur la méthodologie d'estimation des poids à âges types avant sevrage appliquée aux caprins Créoles producteurs de viande. Rev. Elev. Méd. vét. Pays trop., 54 : 81 87.

28. PERRET G., BIBE B., 1979. L'amélioration de la croissance et son contrôle chez les agneaux. In : $5^{\text {es }}$ Journées de la recherche ovine et caprine : I'amélioration génétique des espèces ovine et caprine. Paris, France, Inra/Itovic, p. 386-402.

29. PRUD'HON M., 1976. La croissance globale des agneaux : ses caractéristiques et ses lois. In : $2^{\text {es }}$ Journées de la recherche ovine et caprine. Paris, France, Inra/Itovic, p. 6-26.

30. REKIK M., ALOULOU R., BEN HAMOUDA M., 2005. 2006. Small ruminant breeds of Tunisia. In: Iniguez L. Ed., Characterisation of small ruminant breeds in West Asia and North Africa. Vol. 2: North Africa. Aleppo, Syria, ICARD, p. 91-140.

31. REKIK M., BEN HAMOUDA M., 2000. A steering frame for the genetic improvement of sheep and goat in Tunisia. Cah. Options méditerr. Sér. A, 43 : 129-136.

32. REKIK M., MAHOUACHI M., GHARBI M., ATTIA W., MEDHIOUB L., 2000. Le dilemme de l'élevage ovin extensif dans les régions élevées du Nord-Ouest semi-aride tunisien. Rev. Elev. Méd. vét. Pays trop., 53 : 377-386.

33. RICHARDS F.J., 1959. A flexible growth function for experimental use. J. exp. Bot., 10: 280-300.

34. RICORDEAU G., BOCCARD R., 1961. Relation entre la quantité de lait consommé par les agneaux et leur croissance. Ann. Zoot., 10 : 113125.

35. SAS/STAT, 1996. User Guide, vers. 6.1. Cary, NC, USA, SAS Institute. 36. TOPAL M., OZDEMIR M., AKSAKAL V., YILDIZ N., DORGU U., 2004. Determination of the best nonlinear function in order to estimate growth in Morkaram and Awassi lambs. Small Rumin. Res., 55: 229-232.

Accepté le 10.07.2012 


\section{Summary}

Ben Hamouda M. Growth monitoring of suckling lambs in Tunisia. I. Global curve fitting and critical analysis of the conventional protocol

Growth monitoring data of two experimental sheep herds of Barbarine breed, collected during 31 years on 9642 lambs weighed at birth, then six times at 21-day intervals, were analyzed. Estimates obtained by fitting a global growth curve were compared to assessments carried within the "F2" conventional protocol of growth. Brody, logistic, Gompertz and von Bertalanffy functions were adequately adjusted to the seven controls. The fitting factors, coefficients of determination $\left(R^{2}\right)$ and residual sums of squares were estimated at 0.997 and $2.9,0.997$ and 3.0, 0.996 and 3.8, and 0.996 and 3.8, respectively, for the functions of von Bertalanffy, Gompertz, Logistic and Brody. The average weights at typical ages, estimated by the conventional method, were 3.4, 5.5, 9.1, 15.5, 18.2 and $22.2 \mathrm{~kg}$ at birth, 10, 30, 70, 90 and 120 days of age, respectively. The calculation of performances at typical ages by the conventional method was accompanied by a small loss of precision of $5,2,0.8$ and $0.5 \%$ at $30,70,90$ and 120 days, and by a sizeable drop of 25, 19, 12 and $10 \%$ of DWG10/30 (daily weight gain between 10 and 30 days), DWG30/70, DWG30/90 and DWG30/120, respectively. The loss of precision associated with DWG10/30 exceeded 50\% for the interval birth - first control at 21 days because of a lower and imprecise extrapolation of the weight at 10 days.

Keywords: Sheep - Body weight - Weight gain - Calculation Growth control - Tunisia.

\section{Resumen}

Ben Hamouda M. Control del crecimiento de ovejas lactantes en Túnez. I. Ajuste de una curva global y el análisis crítico del protocolo oficial

Fueron analizados los datos del control de crecimiento de dos rebaños experimentales de ovejas de raza Barbarine, recogidos durante 31 años, referente a 9.642 corderos controlados al nacimiento y posteriormente pesados seis veces en intervalos de 21 días. Las estimaciones obtenidas gracias al ajuste de una curva de crecimiento global se compararon con las evaluaciones realizadas bajo el protocolo convencional de control de crecimiento "F2". Las funciones Brody, logística, Gompertz y von Bertalanffy fueron ajustadas adecuadamente a la serie de siete controles. Los criterios de ajuste, los coeficientes de determinación $\left(R^{2}\right)$ y las sumas de los cuadrados de las diferencias residuales han sido estimados en 0,997 y $2,9,0,997$ y $3,0,0,996$ y 3,8 , y 0,996 y 3,8 , respectivamente, para las funciones de von Bertalanffy, Gompertz, logística y Brody. Los pesos promedios por edad estándar, estimada por el método oficial, fueron de 3,4, 5,5, 9,1, 15,5, 18,2 y 22,2 $\mathrm{kg}$, respectivamente al nacimiento, a los 10, 30, 70, 90 y 120 días de edad. El cálculo de los rendimientos por edad estándar, según el método convencional, fue acompañado de una pequeña pérdida de precisión de los pesos del orden de 5, 2, 0,8 y $0,5 \%$, respectivamente a los 30, 70, 90 y 120 días, y de una disminución considerable de $25,19,12$ y $10 \%$, respectivamente en la GMD10/30 (ganancia media diaria entre 10 y 30 días), GMD30/70, GMD30/90 y GMD30/120. La pérdida de precisión asociada a la GMD10/30 superó el 50\% en el intervalo nacimiento - primer control a los 21 días debido a una extrapolación inferior, imprecisa, del peso a los 10 días.

Palabras clave: Ovino - Peso corporal - Ganancia de peso Cálculo - Control del crecimiento - Túnez. 
